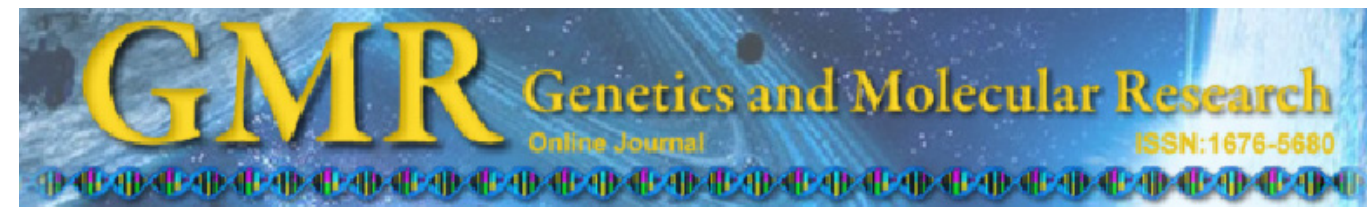

\title{
Successful preimplantation genetic aneuploidy screening in Turkish patients
}

\author{
N. Ercelen ${ }^{1}$, E. Turtar ${ }^{1}$, M. Gultomruk ${ }^{1}$, H. Comert ${ }^{1}$, H. Coskun ${ }^{1}$, \\ R. Mercan' ${ }^{2}$ and A. Nuhoglu ${ }^{2}$ \\ ${ }^{1}$ Genetic Disease Diagnostics Center, Istanbul, Turkey \\ ${ }^{2}$ Assisted Reproduction Unit, American Hospital, Istanbul, Turkey \\ Corresponding author: N. Ercelen \\ E-mail: nesrinercelen@gmail.com
}

Genet. Mol. Res. 10 (4): 4093-4103 (2011)

Received October 7, 2011

Accepted November 10, 2011

Published November 17, 2011

DOI http://dx.doi.org/10.4238/2011.November.17.6

\begin{abstract}
Preimplantation genetic diagnosis is a preventive approach for identifying genetic abnormalities in early stages of reproduction. We used preimplantation genetic aneuploidy screening in 230 cycles of patients with indications of advanced maternal age, recurrent implantation failure, recurrent spontaneous abortions, or severe male factor. Biopsied blastomeres from embryos with six to eight blastomeres on day 3 were fixed and fluorescence in situ hybridization was utilized on chromosomes 13, 16, 18, 21, 22, X, and Y. Among 945 morphologically normal embryos, 314 were diagnosed as chromosomally normal. Trisomy and monosomy were observed in $36 \%$ of the cases (18\% each). Embryo transfer was used in 144 cycles, resulting in 41 pregnancies. Thirty-seven healthy babies were delivered, with a take-home baby rate of $24.2 \%$ and an implantation rate of $22 \%$. We recommend preimplantation genetic aneuploidy screening as a valuable technique to select normal chromosome embryos in order to avoid multiple pregnancies due to the multiple embryo transfers that are normally necessary to ensure pregnancy in poor prognosis in vitro fertilization patients.
\end{abstract}

Key words: PGAS; Fluorescence in situ hybridization; Aneuploidy screening 


\section{INTRODUCTION}

The aim of reproductive genetics is to evaluate the patient or couple prior to fertilization, evaluate embryos at the preimplantation stage and test the fetus in an ongoing pregnancy for a genetic disease or an abnormality in close association with reproductive medicine, assisted reproductive technologies (ART) and developmental genetics. Preimplantation genetic diagnosis (PGD), unlike prenatal testing, screens polar bodies or embryos at the preimplantation stage for genetic diseases or abnormalities that pose a risk for the developing embryo and the probable offspring.

Since its first introduction as a sex determination tool for X-linked diseases (Handyside et al., 1990), PGD has greatly widened its scope of application. A decade after its introduction, PGD now offers solutions for many indications, such as chromosomal abnormalities including translocations, Mendelian disorders, and human leukocyte antigen (HLA) typing for selection of healthy and compatible embryos for transfer. In more than 6000 PGD cycles performed worldwide, approximately 1000 babies were born (Verlinsky et al., 2004). The prevalence of major congenital malformations in these babies (5-6\%) was not different from that observed in the general population (ESHRE PGD Consortium Steering Committee, 2005).

PGD for aneuploidy screening (PGD-AS) is one of the major applications of PGD in couples who could not benefit from IVF treatments (Wilton, 2002). PGD-AS was mainly introduced for the low implantation rates in IVF due to chromosomal aneuploidies. The ease of PGD-AS compared to other PGD applications and the diversity of patient populations and/ or indications available has helped PGD-AS surpass the other indications of PGD (ESHRE PGD Consortium Steering Committee, 2002). ESHRE PGD Consortium Steering Committee (2005) declared PGD-AS as the major indication for PGD.

Advanced maternal age (AMA), recurrent implantation failure (RIF), and recurrent spontaneous abortions (RSA) constituted the three main indications for PGD-AS. ESHRE PGD Consortium Steering Committee (2002) reported 20\% clinical pregnancy rate per oocyte retrieval (OR) among these three indication groups in 801 PGD-AS cycles. The report of the ESHRE PGD Consortium Steering Committee (2005) included pregnancy rates (clinical pregnancy rate per oocyte retrieval) of 18 and $15 \%$ for indications of RSA and RIF, respectively. Same data provided a lower pregnancy rate in AMA group (9\%) which can be partly explained by the relatively low number of oocytes collected in this group.

In this study, we present our results of 230 cycles of PGD-AS that were performed in our center for three major indications of PGD-AS: AMA, RIF and RSA together with severe male factor (SMF).

\section{MATERIAL AND METHODS}

In this study, we analyzed 230 PGD-AS cycles performed in 153 couples referred to the American Hospital Genetic Disease Diagnostics Center between October 2001 and May 2004. Genetic counseling together with information on PGD-AS was given to all couples and a written consent was obtained. Chromosomal analysis was performed from peripheral blood samples of each couple prior to the PGD-AS cycle. The AMA group consisted of patients 38 or older. Patients with three or more previous implantation failures were included in the RIF group. Patients in the RSA group had three or more pregnancy losses. SMF was defined as 
severe oligo/azoospermia, with severe oligospermia defined as a sperm count of less than 1 million $/ \mathrm{mL}$. Patients with multiple indications were grouped according to their primary indication for PGD-AS.

Assisted reproduction techniques including ovarian stimulation, oocyte recovery, IVF or ICSI and culture of embryos for PGD were performed. When the leading follicle reached 20 $\mathrm{mm}$ with two more follicles of $\geq 16 \mathrm{~mm}$ in diameter, final maturation of the oocytes was triggered with 10,000 IU of human chorionic gonadotrophin (HCG; Profasi, Serono) following ovarian stimulation. Thirty hours after HCG administration oocyte recovery was performed. Embryos were individually cultured in microdroplets containing G1 medium on days 1 and 2 and G2 medium on days 3-5 following ICSI or IVF (G1 and G2 media; Vitro Life, IVF Science Scandinavia, Gotenburg, Sweden) (Balaban et al., 2004). Only two pronucleated (2PN) zygotes were taken into account in our study. Blastomere biopsy was performed on embryos on the third day of development according to their blastomere number $(\geq 5)$ and quality $(<20 \%$ fragmentation). Zona pellucida opening was performed by using a $1.48 \mu \mathrm{m}$ infrared diode laser in a computer-controlled system (IVF Work Station and Zona Laser Treatment System, Hamilton Thorne Instruments, Beverly, MA, USA) which is attached to the objective turret of an Olympus $1 \mathrm{X}-70$ inverted microscope. Zona openings of the embryos placed in $5 \mu \mathrm{L}$ droplets of calcium-magnesium-free medium (EB-10; Vitrolife, Gotenburg, Sweden) were achieved by four or five shots of $25 \mathrm{~ms}$ using $55 \mathrm{MW}$ power density. A single anucleated blastomere was removed from each embryo and replaced in $5 \mu \mathrm{L}$ droplets of G-PGD medium. Biopsied blastomeres in G-PGD medium (VitroLife Inc., Sweden) were transferred and washed in hypotonic solution. For the fixation of nuclei, blastomeres were treated with HCI/Tween 20 solution and placed on SuperFrost Plus clean glass slide (Menzel-Gläser, Braunschweig, Germany) under an Olympus CK40 inverted microscope (Olympus Corporation, Tokyo, Japan). Cytoplasmic breakage and removal of cytoplasmic debris were facilitated with another drop of methanol/ acetic acid $(3: 1, \mathrm{v} / \mathrm{v})$ treatment.

After washing of the slides in $2 \mathrm{X} \mathrm{SSC}$ at $37^{\circ} \mathrm{C}$ and formaldehyde treatment, they were placed in pepsin solution to remove residual cytoplasm, which may interfere with FISH signals. This was followed by a $70,85 \%$ and pure ethanol series for dehydration of slides. Specific probe mixtures were added onto the slides and hybridization was accomplished in HYBrite instrument (Vysis Inc., Downers Grove, IL, USA) by a denaturation step at $73^{\circ} \mathrm{C}$ for $5 \mathrm{~min}$ and a hybridization step at $37^{\circ} \mathrm{C}$ for $4 \mathrm{~h}$. As for the second-round hybridization, slides were left under light to bleach the signals left from the first-round hybridization and then placed in methanol solution for $5 \mathrm{~min}$. After $2 \mathrm{X} \mathrm{SSC}$ and formaldehyde treatment, the slides were dehydrated with the same alcohol series and a second probe set was hybridized for 5 min. at $73^{\circ} \mathrm{C}$ and for $16 \mathrm{~h}$ at $37^{\circ} \mathrm{C}$ in HYBrite instrument. MultiVysion PB multicolor probe set (Vysis Inc.) including chromosomes 13, 16, 18, 21, 22 and Cep XY probe set (Vysis Inc.) consisting of probes for chromosomes $\mathrm{X}$ and $\mathrm{Y}$ were used in the first and second round hybridization, respectively. Post hybridization washing was performed in $0.4 \mathrm{X} \mathrm{SSC}, 0.3 \% \mathrm{NP} 40$ for $5 \mathrm{~min}$ and in $2 \mathrm{X} \mathrm{SSC}, 0.1 \% \mathrm{NP} 40$ for $1 \mathrm{~min}$. Slides were dried and Antifade solution (Vysis Inc.) was applied on slides in the first round hybridization. In the second round hybridization, slides were counterstained with DAPI (Vysis Inc.) prior to signal observation. A Nikon E-800 fluorescent microscope (Nikon Corporation, Tokyo, Japan), with appropriate filters for the spectra of the probes was used to detect FISH signals which were evaluated by two biologists according to the scoring criteria described by Munné (2003). FISH images of each nucleus 
were captured and analyzed by using Quips image analysis software (Vysis Inc.).

Embryos regarded as normal by FISH were transferred on day 5. Resulting pregnancies were followed and prenatal diagnosis for each pregnancy was suggested.

Tests were used to compare the results of PGD-AS with respect to three maternal age groups according to clinical outcome. Outcome measures were compared by using variant analysis in all groups. PGD-AS data was statistically analyzed by using the chi-square $\left(\chi^{2}\right)$ test and $2 \times 2$ contingency tables. Significant difference was assigned as $\mathrm{P}<0.05$.

\section{RESULTS}

In 230 PGD cycles, PGD-AS was performed for patients with AMA in 79 (34.3\%), with RIF in $62(27.0 \%)$, with RSA in $28(12.2 \%)$ and with SMF in $34(14.8 \%)$ cycles. Five cycles with karyotype abnormalities and 22 cancelled cycles due to high cost and poor embryo quality constituted 2.2 and $9.6 \%$ of the total 230 cycles, respectively.

The mean maternal age was $36.6 \pm 5.5$ (Table 1). Mean maternal ages of each indication group were $41.2 \pm 2.4$ (min. 33- max. 48) in AMA, $35.2 \pm 4.4$ (min. 25-max. 44) in RIF, $33.7 \pm 4.6$ (min. 25- max. 41) in RSA and $30.9 \pm 4.9$ (min. 23- max. 40) in SMF. The average previous IVF cycles of patients undergoing PGD in this study was $2.8 \pm 2.4$. Among $965 \mathrm{em}-$ bryos analyzed with respect to the chromosomes 13, 16, 18, 21, 22, X and Y, FISH diagnosis was reached in $945(97.9 \%)$. Average number of embryos analyzed per cycle was $5.1 \pm 3.3$. Of those FISH diagnosed embryos, 314 (33.2\%) were normal and $631(66.8 \%)$ were found to have various chromosomal abnormalities. Among these chromosomally abnormal embryos, the most common abnormality was aneuploidy with the gain or loss of one chromosome (Table 1). The highest chromosomal abnormality rate was observed in chromosome $16(20 \%)$, which was followed by sex chromosomes (18.7\%). In monosomic embryos, monosomy 16 was detected with a rate of $22.1 \%$. Complex aneuploidy was observed in $21 \%$ of the abnormal embryos. Polyploid, and haploid embryos constituted 9.6 and $11.5 \%$ of abnormal embryos, respectively. Other abnormalities were detected in $21.9 \%$ of abnormal embryos. FISH diagnosed embryos were transferred with an average transfer number of $1.6 \pm 0.8$ (min. 1- max. 3) per cycle (Table 1).

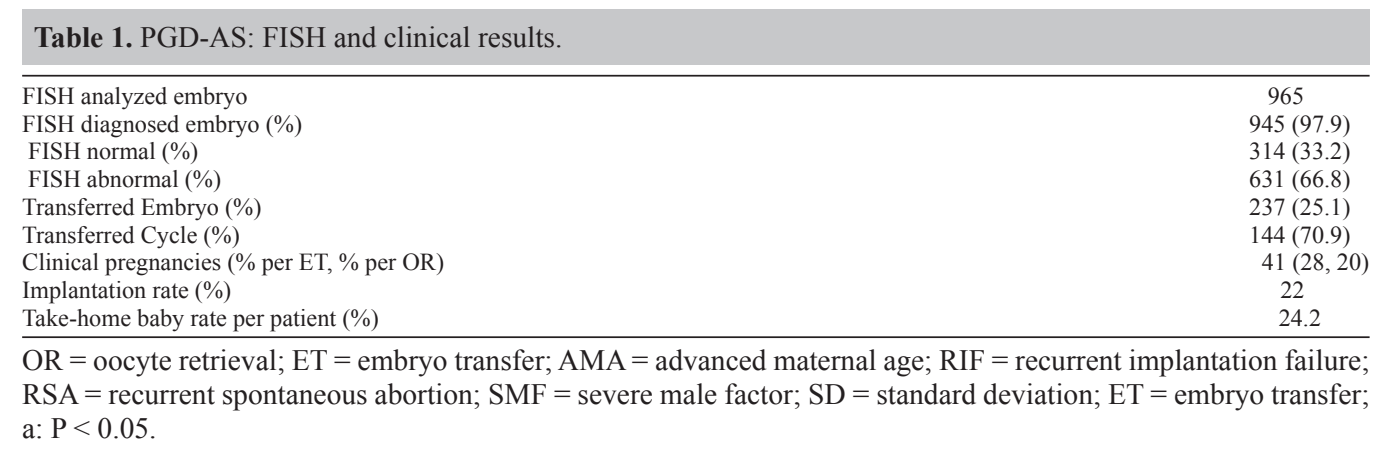

Table 2 represents the comparison of 203 PGD cycles with respect to indication groups. AMA and RIF groups together constituted more than half of the total cycles (61.3\%). In each group, over $97 \%$ of the embryos could be diagnosed by FISH. The highest chromo- 
somal abnormality rate was observed in the RSA group (73.9\%). On the other hand, a higher rate of normal embryos (41.6\%) was obtained from the SMF group when compared to other indication groups $(\mathrm{P}<0.05)$. The relationship between the four indication groups with respect to FISH results is shown in Figure 1. No significant difference was observed in terms of clinical pregnancy and implantation rates among AMA, RIF, RSA and SMF groups $(\mathrm{P}>0.05)$. Figure 2 shows the pregnancy and implantation rates among study groups.

Table 2. The comparison of FISH and clinical results with respect to indications.

\begin{tabular}{lcccc}
\hline Indications & $A M A$ & $R I F$ & $R S A$ & $S M F$ \\
\hline Cycles (\%) & $79(34.3)$ & $62(27.0)$ & $28(12.2)$ & $34(14.8)$ \\
Mean maternal age (Mean \pm SD) & $41.2 \pm 2.4$ & $35.2 \pm 4.4$ & $33.7 \pm 4.6$ & 158 \\
Analyzed embryo (n) & 287 & 339 & 97.5 & 26.1 \\
Diagnosed embryo (\%) & 97.9 & 97.6 & 73.9 & 98.9 \\
FISH normal (\%) & 32.9 & 32.6 & 35.0 & $41.6^{\mathrm{a}}$ \\
FISH abnormal (\%) & 67.1 & 67.4 & 25.0 & 38.4 \\
Clinical pregnancy rate (\%/ET) & 23.1 & 21.0 & 25.1 \\
Clinical pregnancy rate (\%/OR) & 15.2 & 25.3 & 25.7 & 20.8 \\
Implantation rate (\%) & 17.7 & & &
\end{tabular}

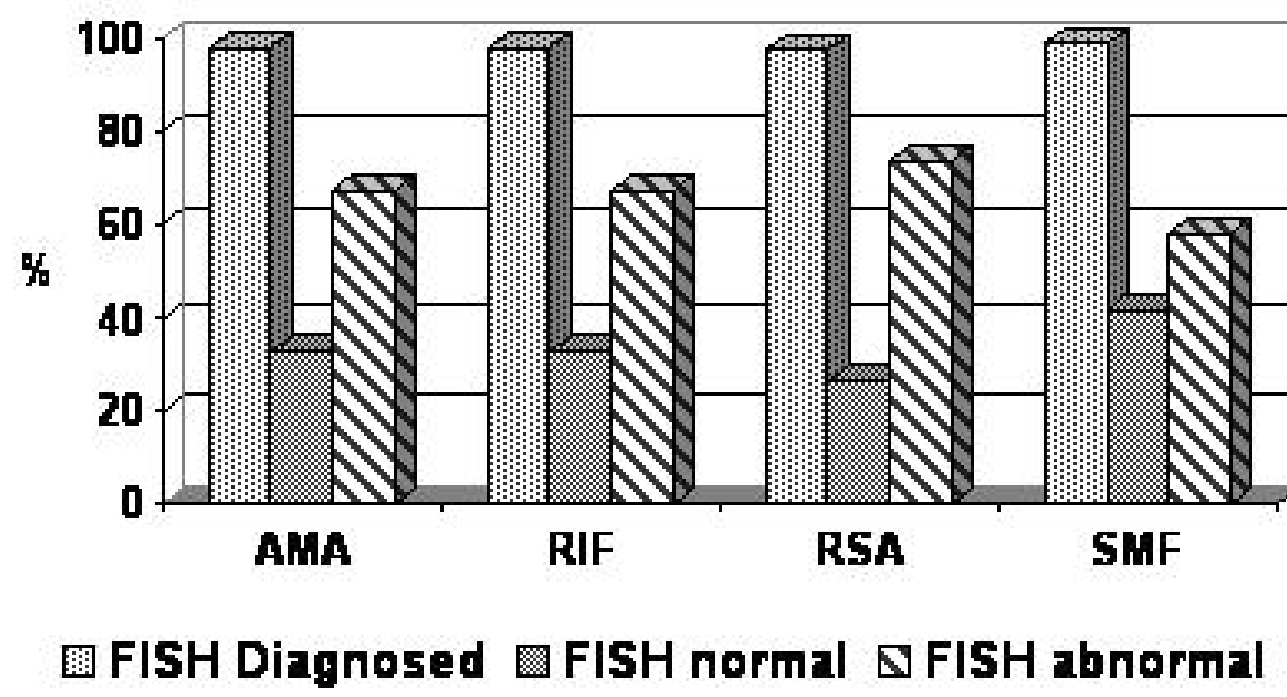

Figure 1. FISH results with respect to indication groups.

Overall, in 203 cycles, 41 clinical pregnancies with rates of 28 (clinical pregnancy/ ET) and 20\% (clinical pregnancy/oocyte retrieval) were obtained and 37 healthy babies were delivered. The implantation rate among all indication groups was $22 \%$. Take-home baby rate per patient was calculated as $24.2 \%$. Prenatal diagnosis of each pregnancy was also performed and no abnormality was detected confirming PGD-AS results. Pregnancy outcomes were also followed and no congenital malformations or neonatal complications were observed in any of the babies delivered. 
We evaluated PGD-AS outcome with respect to maternal age intervals (Table 3). Patients aged between 30 and 48: patients aged between 30-35; between 36-40 and patients aged 40 or above. The rate of chromosomally abnormal embryos was increased as the age advanced non-significantly, whereas a significant decrease in pregnancy rates $(11.4$ and $7.5 \%$ as clinical pregnancy/ET and /OR, respectively) was observed in patients older than $40(\mathrm{P}<0.05)$. The implantation rate $(7.6 \%)$ of these patients was also significantly lower when compared to other age groups $(\mathrm{P}<0.05)$. We could not detect any significant difference in terms of pregnancy and implantation rates among the other two groups (30-35 and 36-40). However, euploidy rates of the embryos decreased gradually and significantly with increasing maternal age.

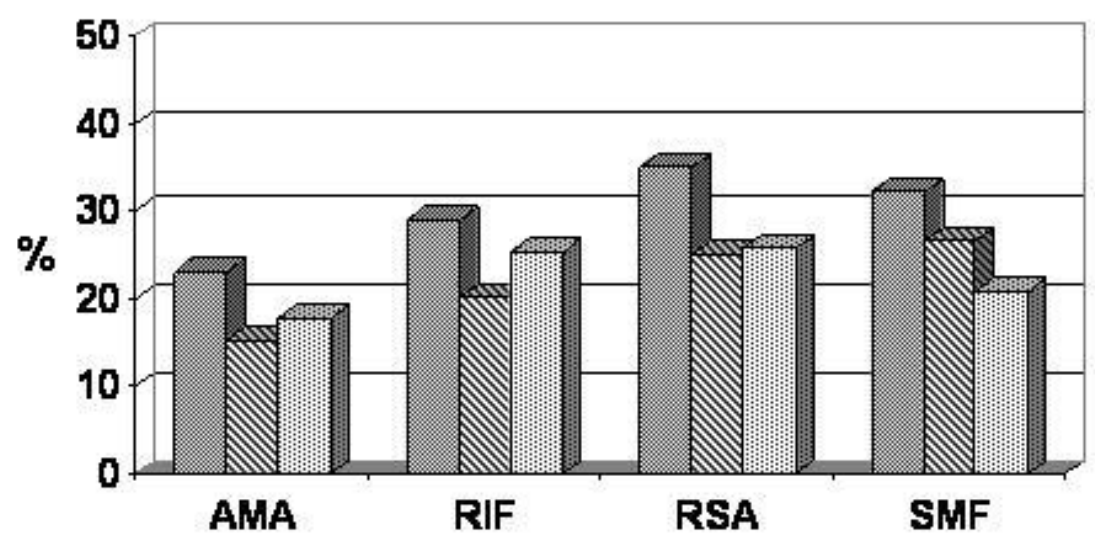

\section{Dregnancy rate (IET) \& Pregnancy rate (IOR) 圈 Implantation rate}

Figure 2. Pregnancy and implantation rates among study groups.

\begin{tabular}{|c|c|c|c|}
\hline Maternal age, years & $30-35$ & $36-40$ & over 40 \\
\hline Cycle & 48 & 64 & 67 \\
\hline Mean maternal age (Mean $\pm \mathrm{SD}$ ) & $33.1 \pm 1.4$ & $38.0 \pm 1.5$ & $42.2 \pm 1.7$ \\
\hline FISH analyzed embryos (n) & 327 & 314 & 256 \\
\hline FISH normal (\%) & $110(40.3)$ & $98(37.5)$ & $69(34.5)$ \\
\hline FISH abnormal (\%) & $163(59.7)$ & $163(62.5)$ & $131(65.5)$ \\
\hline \multicolumn{4}{|l|}{ Clinical pregnancy rate $(\%)$} \\
\hline Clinical pregnancy/OR & 29.2 & 23.4 & $7.5^{\mathrm{a}}$ \\
\hline Clinical pregnancy/ET & 35.9 & 34.9 & $11.4^{\mathrm{a}}$ \\
\hline Implantation rate (\%) & 24.2 & 23.9 & $7.6^{\mathrm{a}}$ \\
\hline
\end{tabular}

$\mathrm{OR}=$ oocyte retrieval; $\mathrm{ET}=$ embryo transfer; $\mathrm{a}: \mathrm{P}<0.05$.

\section{DISCUSSION}

PGD-AS is used as a selection tool for chromosomally normal embryos in poor prognosis IVF patients. The patient spectrum of PGD-AS has been widened and the indication groups for PGD-AS have been determined by the understanding of chromosomal abnormalities observed in early human embryos and spontaneous abortions. In our study, patients in 
AMA, RIF, RSA and SMF groups underwent PGD-AS for screening of chromosomes 13, 16, $18,21,22, \mathrm{X}$ and $\mathrm{Y}$ in 203 PGD cycles.

High levels of aneuploidy (70\%) in human embryos were shown by Munné (2003). High percentages of abnormal embryos $(\sim 65 \%)$ detected by FISH were also reported for at least one of the chromosomes studied in patients with indications for PGD-AS (Gianaroli et al., 2005). Another study has also demonstrated significantly higher incidence of chromosomal abnormalities $(71 \%)$ in embryos of non-pregnant patients when compared to those of pregnant patients after PGD (55\%) (Taranissi et al., 2005). Apart from the studies on human blastomeres, Verlinsky et al. (1995) analyzed the first and/or second polar bodies of oocytes by using FISH probes for combinations of chromosomes 13, 18, 21 and $\mathrm{X}$ and demonstrated an abnormality rate of $23.2 \%$ among FISH diagnosed oocytes. Our FISH analysis of $965 \mathrm{em}-$ bryos revealed that $66.8 \%$ of the embryos had chromosomal abnormalities in which complex aneuploidy and monosomy-trisomy were common. Although similar results were obtained when compared to previous studies, differences in the level of abnormalities can be attributable to the nature of the patient population undergoing PGD-AS. The number of chromosomes studied is also of great importance. Screening a higher number of chromosomes (e.g., inclusion of chromosomes 15, 16 and 22) might increase the rate of abnormality observed (Gianaroli et al., 2005).

Several groups have also investigated the effect of PGD-AS on pregnancy and implantation rates (Munné, 2003). International Working Group on PGD (2001) reported a 24\% pregnancy rate through 3000 PGD cycles and data collection I-III of ESHRE PGD Consortium included an overall pregnancy rate (clinical pregnancy/OR) of $20 \%$ in PGD-AS patients. A multicenter report consisting of 3747 PGD-AS cycles provided a $23.3 \%$ pregnancy rate per embryo transfer (Verlinsky et al., 2004). As for implantation rates, Gianaroli et al. (2005) studied 3-day embryos and in that prospective study, PGD-AS resulted in an implantation rate of $28 \%$ which was higher compared to that of $12 \%$ in control patients. In our study, we obtained an overall pregnancy rate of $28 \%$ (clinical pregnancy/embryo transfer) and an implantation rate of $22 \%$. We did not observe any significant difference among indication groups in terms of pregnancy and implantation rates. The PGD-AS outcome of our study is in accordance with previous studies. The rate of embryo transfer also affects the PGD-AS outcome. In our study, embryo transfer was not achieved in $58(29.6 \%)$ cycles due to lack of chromosomally normal embryos. We have also obtained a take-home baby rate of $24.2 \%$, which was similar to that of Gianaroli et al. (2005), who compared the take-home baby rates in patients with previous unsuccessful obstetric history undergoing PGD and reported a significantly higher take-home baby rate $(23.1 \%)$.

Munné (2003) demonstrated the association between maternal age and the competence of the embryo as aneuploidy. Our FISH results in the AMA group also indicated a high rate of chromosomally abnormal embryos (67.1\%). The study by Gianaroli et al. (2005) concluded that by selecting the euploid embryos with respect to the chromosomes screened, the age factor in AMA patients aged between 36 and 42 can be obviated. Munné (2003) also obtained a significant increase in the implantation rate (17.6\%) after PGD when compared to $10.6 \%$ in the matched control group. In our AMA group consisting of women aged between 33 and 48, an overall pregnancy rate (clinical pregnancy/embryo transfer) of $23.1 \%$ and an implantation rate of $17.7 \%$ were obtained. Factors such as involvement of abnormalities of other chromosomes, oocyte aging and uterine receptivity should also be taken into consideration in 
women of older ages.

It was reported that women aged 37 or older with at least six embryos can benefit most from PGD-AS. (Munné, 2003). In our study, significantly lower pregnancy and implantation rates were observed in patients aged above 40, when compared to women aged below 40 irrespective of the indication. This can be explained by low quality oocytes mostly due to chromosomal abnormalities and poor implantation factors in aged patients. There was also an increase in pregnancy rates of patients aged between 36-40, who had more than 6 embryos when compared to that of patients in the same age group, but had less than 5 embryos (unpublished data) which was supported by the outcome of a previous study (Munné, 2003).

Gianaroli (2005) observed no statistical difference in pregnancy and implantation rates of patients, who did not benefit from at least three previous IVF cycles due to implantation failure. Other studies also showed no direct benefit of PGD in patients with recurrent implantation failure (Werlin, 2003). In our RIF group, we obtained a pregnancy rate (clinical pregnancy/embryo transfer) of $28.9 \%$ and an implantation rate of $25.3 \%$ with an abnormal embryo rate of $67.4 \%$, which were close to other reports (ESHRE PGD Consortium Steering Committee, 2002). Besides the chromosomal aneuploidies detected by FISH, other chromosomal abnormalities may also contribute to poor IVF outcome in RIF patients. Cytoplasmic transfer in RIF patients showed improved pregnancy and implantation rates (Barritt et al., 2001), which indicates the existence of other abnormalities in egg cytoplasm of RIF patients. Non-chromosomal factors such as hormonal or immunological characteristics can also play a role in the low success of PGD-AS in RIF patients. Other factors affecting the PGD-AS outcome might be problems related with the female reproductive system, such as endometrial receptivity. Taranissi et al. (2005) reported poor PGD-AS outcomes in patients aged above 41 , with recurrent implantation failure, further indicating the effect of increased maternal age on this group of patients. Prospective randomized studies and inclusion of the effect of other factors mentioned are necessary to determine and improve the success of PGD-AS in patients with RIF. However, PGD-AS can be used to identify the reasons behind recurrent implantation failure as previously mentioned by Caglar et al. (2005) and to guide couples and physicians for possible reproductive options.

Studies of PGD-AS in patients with recurrent spontaneous abortions revealed that aneuploidy is a common cause of spontaneous abortions (Munné, 2003). Moreover, PGD-AS was reported to reduce the risk of trisomic offspring, increase implantation rates, and decrease spontaneous abortions (Munné, 2003). In the study by Gianaroli et al. (2005), embryos assessed as normal by PGD-AS for chromosomes 13, 18, 21, X and $\mathrm{Y}$ were transferred and this group obtained a $28 \%$ implantation rate, which was higher than that of $12 \%$ in control patients. Munné (2003) studied chromosomes 13, 14, 15, 16, 18, 21, 22, X and Y, which constituted $40 \%$ of all chromosome content, and transfer of normal embryos resulted in $50 \%$ pregnancy rate per transfer, although the sample size was small $(\mathrm{N}=25)$. Through studying a larger group of patients, a $23 \%$ implantation rate together with a $33 \%$ ongoing pregnancy rate was reported (Gianaroli et al., 2005). Gianaroli et al. (2005) obtained $\sim 30 \%$ pregnancy rate with a high percentage (66\%) of chromosomally abnormal embryos. Rubio et al. (2005) also observed a higher percentage of abnormal embryos and lower pregnancy and implantation rates in the RSA group, when compared to patients with sex-linked diseases as controls. A prospective cohort study implied that there is no direct impact of PGD-AS on RSA patients irrespective of the maternal age (Platteau et al., 2005). Our group observed the highest abnormality rate in 
RSA patients (73.9\%). The rates of pregnancy (clinical pregnancy/embryo transfer) (35\%) and implantation (25.7\%) obtained were in harmony with previous studies and PGD-AS can be regarded as a beneficial tool for patients with RSA. The outcome of PGD-AS might be improved by identification of new chromosomal abnormalities involved in spontaneous abortions and subsequent screening of them. It is clear that by decreasing the abortion rates, take-home-baby rates can be increased. Munné (2003) reported a decrease in spontaneous abortions from $23 \%$ in controls to $9 \%$ in the PGD-AS group which was accompanied by a significant increase in ongoing and delivered babies in the latter group (16\%) when compared to that in the former $(11 \%)$. Apart from the three main indication groups for PGD-AS, we also investigated the effect of PGD-AS in patients with severe male factor. This group of patients had the lowest percentage of chromosomally abnormal embryos (58.4\%) and showed statistical difference when compared to other groups. A high pregnancy rate (32.1\%) was observed in this category; however, the implantation rate $(20.8 \%)$ was lower than RIF and RSA groups. Gianaroli et al. (2003) also reported high pregnancy (20\%) and implantation (14.1\%) rates in the SMF group. The contribution of the male gamete to the chromosomal complement of the embryo is obvious. Therefore, it is expected that sperm carrying chromosomal abnormalities can also affect the chromosomal state of the embryo and consequently the PGD-AS outcome. Higher aneuploidy rates $(79 \%)$ were observed in sperm samples of patients with severe male factor condition (Gianaroli et al., 2005). Kahraman et al. (2000) also reported an aneuploidy rate of $76 \%$ through FISH in patients with morphologically abnormal spermatozoa. Most of the aneuploidies in severe male infertility condition were observed in sex chromosomes (Liebaers et al., 1995). The rate of aneuploidy in sperm has also been shown to increase with the severity of the male factor condition (Gianaroli et al., 2000). The investigation of abnormalities in male gamete might help to explain the success of PGD-AS in this group of patients regardless of the problems related with the female partner.

There is a growing consensus on decreasing the number of embryos transferred into uterus in conventional IVF cycles; however, the number still remains high when compared to that in PGD-AS which was an average of 1.6 in our study. The transfer of a high number of good quality embryos through morphological evaluation increases the rate of multiple pregnancies which in turn results in serious complications affecting the mother and the fetuses (Luke and Keith, 1992; Rao et al., 2004). By employing PGD-AS, besides the traditional morphological criteria, embryos are also screened for their chromosomal make-up and then transferred, decreasing the rate of multiple pregnancies due to the low number of chromosomally normal embryos transferred. This approach also decreases the complications of multiple pregnancies and can be used as an invaluable tool, even to select the best embryo for a single transfer as the technology of PGD-AS further develops in the near future.

The FISH method currently enables the screening of 9 chromosomes simultaneously after two-three rounds of hybridization which constitute $\sim 40 \%$ of all chromosomes. However, abnormalities in other chromosomes or even those that cannot be detected by FISH might be present in patients undergoing PGD-AS and this can lead to implantation failures and spontaneous abortions. By applying comparative genomic hybridization (CGH) for PGD-AS (Voullaire et al., 2000) aneuploidy and mosaicism can be detected for the whole set of chromosomes. Wilton et al. (2003) reported that in RIF patients, approximately $50 \%$ of the embryos diagnosed as normal by FISH revealed to be abnormal when CGH was applied. Abnormalities in every chromosome region larger than $10 \mathrm{Mb}$ in size can be detected without the need 
for preparation of metaphase chromosomes from the sample. However, the relative length of time that the CGH technique requires (3 days for hybridization) and problems related with the amplification of the whole genome has restricted its wide use in PGD centers (Wells and Delhanty, 2000). The use of microarrays with greater resolution $(200 \mathrm{~Kb}-1 \mathrm{Mb})$ has the capacity to open a new era in PGD-AS by providing informative and reliable information regarding the diagnosis of common genetic diseases or chromosomal abnormalities genome-wide.

Abnormal embryo transfer can be avoided by selecting and transferring embryos regarded as normal, ascertained by performing PGD-AS on poor prognosis IVF/ICSI patients. Meanwhile, evaluation of infertility, determination of risks for transmitting a genetic disease to the offspring require an individualized genetic approach to avoid problems in patients undergoing assisted reproductive technologies (ART). Therefore, we propose that PGD can also act as a preventive genetic diagnosis tool for patients undergoing ART treatments to prevent multiple pregnancies and clinical pregnancy termination due to affected offspring.

\section{REFERENCES}

Balaban B, Yakin K, Urman B, Isiklar A, et al. (2004). Pronuclear morphology predicts embryo development and chromosome constitution. Reprod. Biomed. Online 8: 695-700.

Barritt J, Willadsen S, Brenner C and Cohen J (2001). Cytoplasmic transfer in assisted reproduction. Hum. Reprod. Update 7: 428-435.

Caglar GS, Asimakopoulos B, Nikolettos N, Diedrich K, et al. (2005). Preimplantation genetic diagnosis for aneuploidy screening in repeated implantation failure. Reprod. Biomed. Online 10: 381-388.

ESHRE (2002). ESHRE Preimplantation Genetic Diagnosis Consortium: data collection III (May 2001). Hum. Reprod. 17: $233-246$

Gianaroli L, Magli MC, Cavallini G, Crippa A, et al. (2005a). Frequency of aneuploidy in sperm from patients with extremely severe male factor infertility. Hum. Reprod. 20: 2140-2152.

Gianaroli L, Magli MC, Ferraretti AP, Tabanelli C, et al. (2005b). The beneficial effects of preimplantation genetic diagnosis for aneuploidy support extensive clinical application. Reprod. Biomed. Online 10: 633-640.

Griffin DK, Handyside AH, Harper JC, Wilton LJ, et al. (1994). Clinical experience with preimplantation diagnosis of sex by dual fluorescent in situ hybridization. J. Assist. Reprod. Genet. 11: 132-143.

Handyside AH, Kontogianni EH, Hardy K and Winston RM (1990). Pregnancies from biopsied human preimplantation embryos sexed by Y-specific DNA amplification. Nature 344: 768-770.

International Working Group on Preimplantation Genetics (2001). Preimplantation genetic diagnosis-experience of three thousand clinical cycles. Report of the 11th Annual Meeting of the International Working Group on Preimplantation Genetics, in conjunction with 10th International Congress of Human Genetics, Vienna, May 15, 2001. Reprod. Biomed. Online 3: 49-53.

Kahraman S, Bahce M, Samli H, Imirzalioglu N, et al. (2000). Healthy births and ongoing pregnancies obtained by preimplantation genetic diagnosis in patients with advanced maternal age and recurrent implantation failure. Hum. Reprod. 15: 2003-2007.

Liebaers I, Bonduelle M, Van Assche E, Devroey P, et al. (1995). Sex chromosome abnormalities after intracytoplasmic sperm injection. Lancet 346: 1095.

Luke B and Keith LG (1992). The contribution of singletons, twins and triplets to low birth weight, infant mortality and handicap in the United States. J. Reprod. Med. 37: 661-666.

Munné S (2003). Preimplantation genetic diagnosis and human implantation - a review. Placenta 24 (Suppl B): S70-S76.

Platteau P, Staessen C, Michiels A, Van Steirteghem A, et al. (2005). Preimplantation genetic diagnosis for aneuploidy screening in patients with unexplained recurrent miscarriages. Fertil. Steril. 83: 393-397.

Rao A, Sairam S and Shehata H (2004). Obstetric complications of twin pregnancies. Best Pract. Res. Clin. Obstet. Gynaecol. 18: 557-576.

Rubio C, Pehlivan T, Rodrigo L, Simon C, et al. (2005). Embryo aneuploidy screening for unexplained recurrent miscarriage: a minireview. Am. J. Reprod. Immunol. 53: 159-165.

Taranissi M, El-Toukhy T, Gorgy A and Verlinsky Y (2005). Influence of maternal age on the outcome of PGD for aneuploidy screening in patients with recurrent implantation failure. Reprod. Biomed. Online 10: 628-632.

Genetics and Molecular Research 10 (4): 4093-4103 (2011) 
Verlinsky Y, Cohen J, Munne S, Gianaroli L, et al. (2004). Over a decade of experience with preimplantation genetic diagnosis: a multicenter report. Fertil. Steril. 82: 292-294.

Voullaire L, Slater H, Williamson R and Wilton L (2000). Chromosome analysis of blastomeres from human embryos by using comparative genomic hybridization. Hum. Genet. 106: 210-217.

Weier H, Munne S, Lersch RA, Hsieh H, et al. (2001). Towards a full karyotype screening of interphase cells: 'FISH and chip' technology. Mol. Cell Endocrinol. 183 (Suppl 1): S41-S45.

Werlin L, Rodi I, DeCherney A, Marello E, et al. (2003). Preimplantation genetic diagnosis as both a therapeutic and diagnostic tool in assisted reproductive technology. Fertil. Steril. 80: 467-468.

Wilton L, Voullaire L, Sargeant P, Williamson R, et al. (2003). Preimplantation aneuploidy screening using comparative genomic hybridization or fluorescence in situ hybridization of embryos from patients with recurrent implantation failure. Fertil. Steril. 80: 860-868. 